\title{
Simplified analysis of pathogenic leptospiral serovars by random amplified polymorphic DNA fingerprinting
}

\author{
B. G. CORNEY, JANE COLLEY* and G. C. GRAHAM $\dagger$
}

Rockhampton Veterinary Laboratory, Department of Primary Industries, Box 6014 Rockhampton Mail Centre, Queensland 4702, *Animal Research Institute, Department of Primary Industries Locked Mail Bag 4, Moorooka, Queensland 4105 and + Cooperative Centre for Tropical Pest Management, University of Queensland, St Lucia, Queensland 4072, Australia

\begin{abstract}
A rapid, simplified procedure combining random amplified polymorphic DNA (RAPD) fingerprinting of boiled cultures with high resolution agarose gel electrophoresis was used to compare strains from 46 pathogenic leptospiral serovars. The serovars were placed in eight groups on the basis of RAPD profile similarities. Groups 1-7 corresponded with the genome species Leptospira interrogans, $L$. borgpetersenii, $L$. santarosai, $L$. noguchii, $L$. weilii, $L$. kirschneri and $L$. meyeri. The eighth group did not correspond with a known genome species and may represent a new genome species. Primer choice determined the degree of discrimination possible between closely related serovars and genotypes. This procedure, unlike other procedures used for analysing taxonomic relationships between leptospiral serovars, does not require extensive DNA purification, polyacrylamide gel electrophoresis or autoradiography.
\end{abstract}

\section{Introduction}

Random amplified polymorphic DNA (RAPD) fingerprinting [1], also known as arbitrarily primed polymerase chain reaction (AP-PCR) [2] is a polymerase chain reaction (PCR)-based DNA fingerprinting method. RAPD has been used to type isolates of a number of bacteria [3-6]. More recently RAPD was used to determine the taxonomic relationships between leptospiral serovars [7] and for typing bovine isolates of Leptospira [8]. Pérolat et al. [9] used AP-PCR to examine genetic variability among strains of Leptospira serovar hardjo.

Pathogenic serovars of the genus Leptospira are assigned to one of the genome species $L$. interrogans, L. weilii, L. borgpetersenii, L. noguchii, L. santarosai, $L$. inadai or $L$. kirschneri by DNA hybridisation [10, 11]. The non-pathogenic serovars are similarly distributed among several genome species [12]. Ralph et al. [7] used RAPD as a rapid method for classifying serovars. They performed RAPD on purified DNA and analysed the products by polyacrylamide gel electrophoresis (PAGE) followed by autoradiography. This study demonstrates a simpler and more rapid method

Received 30 Dec. 1996; revised version accepted 7 April 1997.

Corresponding author: Dr B. G. Corney. of examining the taxonomic relationships between serovars in which RAPD is performed on boiled cultures and the products are analysed by high resolution agarose gel electrophoresis. This procedure was used by Corney et al. [8] for serovar identification of bovine Leptospira isolates. It has none of the hazards associated with PAGE or autoradiography due to handling acrylamide or radioisotopes.

\section{Materials and methods}

\section{Bacterial strains}

The bacterial strains (Table 1) were obtained from $\mathrm{L}$. Smythe (WHO Collaborating Centre for Reference and Research on Leptospirosis; Brisbane, Queensland, Australia) except for $L$. borgpetersenii serovar hardjobovis strains 11135 and $\mathrm{Hb}-15 \mathrm{~b}-067$, which were obtained from the Commonwealth Serum Laboratories (Parkville, Victoria, Australia) and Dr C. Bolin (National Animal Disease Center, Ames, Iowa, USA), respectively.

\section{$R A P D$}

DNA for RAPD was prepared by rapid lysis of pelleted cells [8]. RAPD was performed with primers L10 and US as described by Corney et al. [8], and primers L7 (5'TGCACCCTCCCCCCGGCCGA3'), L9 (5'TCGCG- 
CTAGAGATGGCGGGG3'), L11 (5'GGCCGGGGGGAGGGTGCAC3') and L12 (5'AGGCGGCGCCAGCAGCGCG3').

\section{Agarose gel electrophoresis}

Up to $20 \mu \mathrm{l}$ of RAPD products were loaded on to agarose $2 \%$ submarine gels by either 'conventional' or 'dry' gel loading methods and electrophoresed for $2 \mathrm{~h}$ at $100 \mathrm{~V}$ (Pharmacia GNA100 electrophoresis cell) or $150 \mathrm{~V}$ (Pharmacia GNA200 electrophoresis cell). Gels were stained with ethidium bromide and photographed. RAPD profiles were compared by eye.

For 'conventional' loading, gel loading buffer consisting of bromphenol blue $0.25 \% \mathrm{w} / \mathrm{v}$, xylene cyanol $0.25 \% \mathrm{w} / \mathrm{v}$ and sucrose $40 \% \mathrm{w} / \mathrm{v}$ [13] was diluted 1 in 6 in RAPD reaction mixture. Gels were submerged in electrophoresis buffer $(0.5 \times \mathrm{TBE} ; 0.045 \mathrm{M}$ Trisborate and $0.001 \mathrm{M}$ ethylenediamine tetraacetic acid disodium salt) [13] and the mixture was loaded through the buffer into the wells. For 'dry loading', horizontal gels were cast as normal and were placed in the electrophoresis cell. Electrophoresis buffer was added to the cell until it was almost lapping the upper gel surface. Each sample was loaded into the dry wells without gel loading buffer, and voltage was applied for 1-2 min. The gels were submerged in buffer and electrophoresis was continued for $2 \mathrm{~h}$.

\section{Results \\ Comparison of 'dry' loading and 'conventional' gel loading}

Preliminary results suggested that 'dry' loading gives clearer profiles with sharper bands than 'conventional' gel loading. To demonstrate the difference, duplicate agarose $2 \%$ gels were loaded with the same volumes of two RAPD reactions and run under identical conditions. 'Dry' loading was used for one gel, and 'conventional' loading was used for the other. 'Dry' loading gave better definition of bands than did 'conventional' loading. Faint bands were clearer and close bands were better resolved. 'Dry' loading was adopted for all RAPD experiments.

\section{Analysis of serovars with primers L10 and US}

Type strains from 45 serovars (as listed by Faine [14]), and two serovar hardjobovis strains, representing 21 serogroups, were analysed by RAPD with primers L10 and US. Each RAPD was performed up to three times. Thirteen serovars were from serogroup Pyrogenes.

Generally, each strain had a unique RAPD profile with each primer. Profiles were reproducible, although there were minor variations in band intensity in some cases. Representative profiles are shown in Figs. 1 and 2. The RAPD profiles were compared by eye, and the strains were placed in RAPD similarity groups $1-8$ as shown in Table 1. Both primers yielded the same groupings. Groups 1-7 correlated with $L$. interrogans, L. borgpetersenii, L. santarosai, L. noguchii, L. weilii, L. kirschneri and L. meyeri, respectively, according to published genome species assignments $[10,11,14]$. Thirteen serovars from serogroup Pyrogenes were distributed among five RAPD groups. The reference strain of serovar hamptoni (also from serogroup Pyrogenes) bore no resemblance to any other serovar, and was the only member of RAPD group 8 .

The type strains of serovars ballum and arborea were exceptions to the above. They had identical profiles with primer L10, but differed by one band with primer US. Similarly, serovars pomona and kennewicki had identical profiles with primers L10 and US; serovars icterohaemorrhagiae and copenhageni were identical with primer L10 and similar with primer US as were serovars zanoni and robinsoni; and serovars hardjobovis and balcanica were similar with both primers.

\section{Resolution of closely related serovars}

As RAPD with primers L10 and US failed to resolve certain serovars, and yielded very similar profiles for others, additional primers L7, L9, L11 and L12 were tested on a selection of these serovars, i.e., pomona, kennewicki, robinsoni, zanoni, balcanica, and genotypes A (strain 11135) and B (strain Hb-15b-067) of hardjobovis [15]. The latter two strains differ by one band with primer L10 and are identical with US [8]. Primers L9, L11 and L12 failed to distinguish between pomona and kennewicki and were discarded. Primer L7 differentiated between the five strains (Fig. 3).

\section{Discussion}

RAPD on crude cell lysates generated specific and reproducible fragment profiles on agarose gels which were characteristic of each leptospiral serovar. Serovars could be assigned to groups that matched each genome species of Leptospira on the basis of overall pattern similarities.

This procedure has several advantages over other similar methods. Rigorous DNA purification is not required. 'Dry' loading the agarose gels gives them sufficient resolution and sensitivity to remove the need for PAGE and autoradiography and the associated hazards. Pattern matching is done by eye, obviating the need for sophisticated computer-based analysis systems. Thus, the technique allows presumptive genome species assignments or isolate typing with minimal capital outlay and minimal exposure to dangerous chemicals.

The fragment profiles were reproducible despite the use of non-standardised crude lysates in the RAPD 

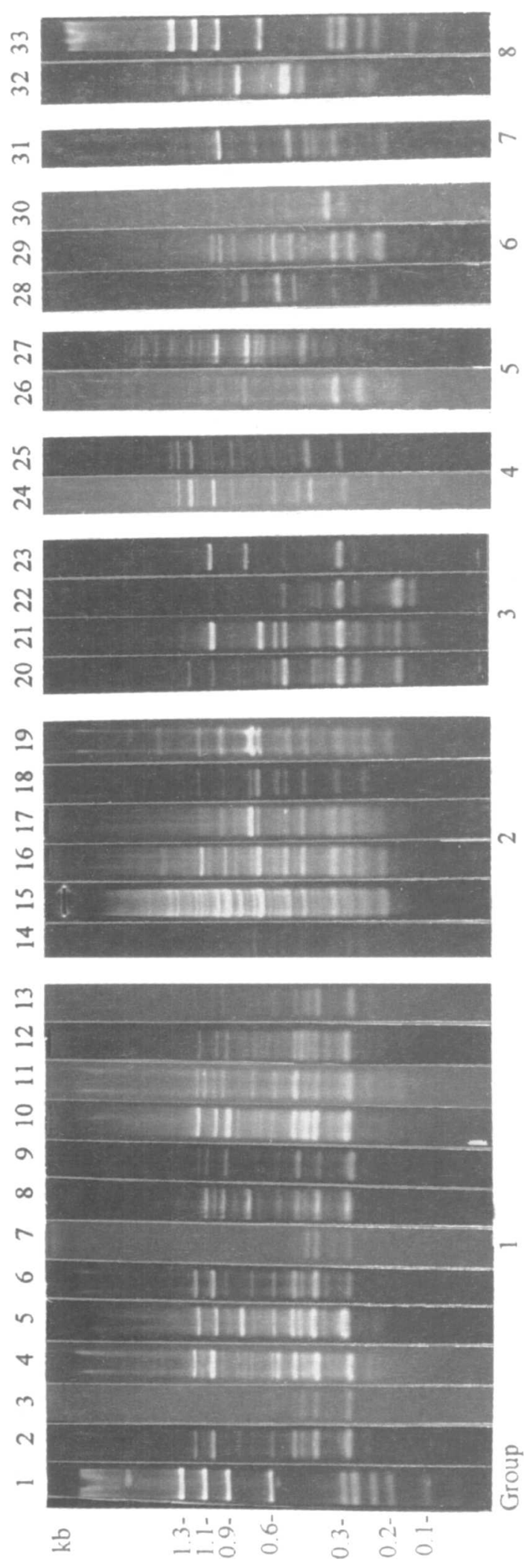

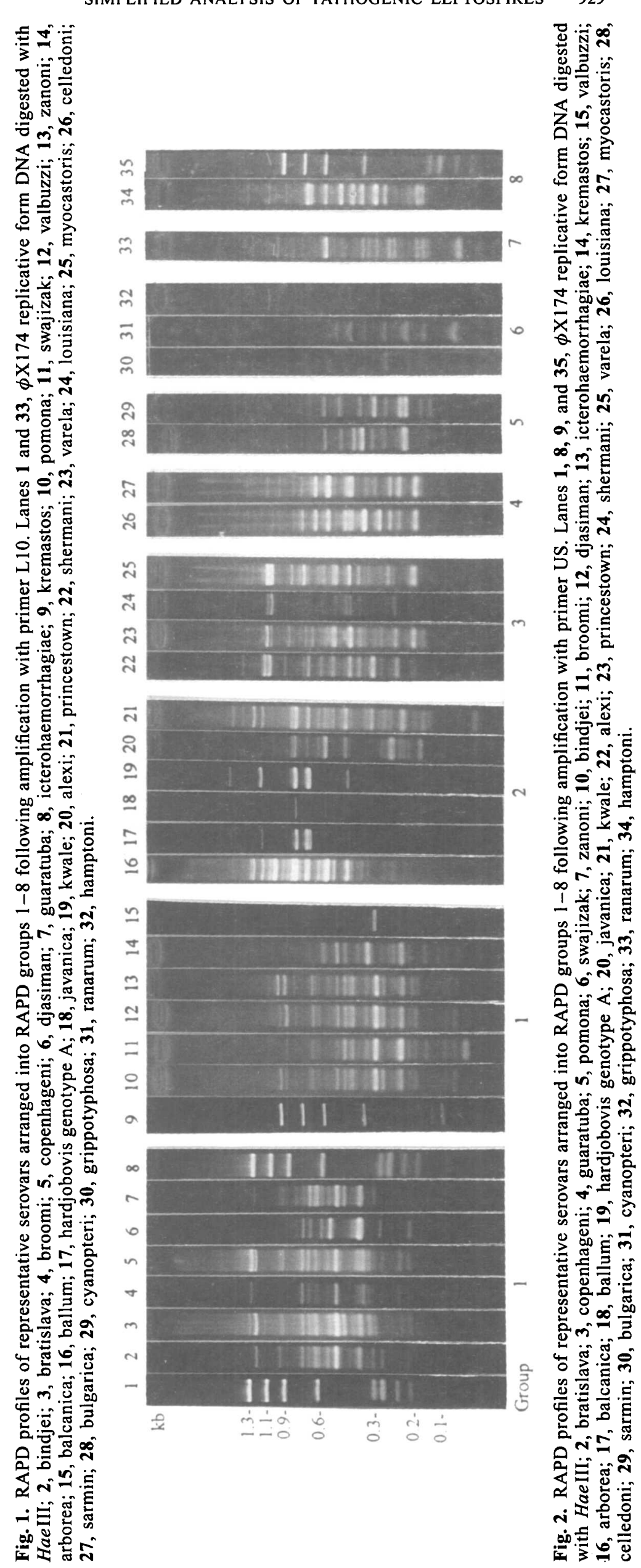


Table 1. Strains of Leptospira used in this study with their genome species and RAPD group assignments

\begin{tabular}{|c|c|c|c|c|}
\hline Serovar & Strain & Serogroup & Genome species* & RAPD group \\
\hline abramis & Abraham & Pyrogenes & L. interrogans & 1 \\
\hline australis & Ballico & Australis & L. interrogans & 1 \\
\hline autumnalis & Akiyami A & Autumnalis & L. interrogans & 1 \\
\hline bataviae & Swart & Bataviae & L. interrogans & 1 \\
\hline biggis & Biggs & Pyrogenes & L. interrogans & 1 \\
\hline bindjei & Bindjei & Canicola & L. interrogans & 1 \\
\hline bratislava & Jež-bratislava & Australis & L. interrogans & 1 \\
\hline broomi & Patane & Canicola & L. interrogans & 1 \\
\hline camlo & LT $64-67$ & Pyrogenes & L. interrogans & 1 \\
\hline canicola & Hond Utrecht IV & Canicola & L. interrogans & 1 \\
\hline copenhageni & M 20 & Icterohaemorrhagiae & L. interrogans & 1 \\
\hline djasiman & Djasiman & Djasiman & L. interrogans & 1 \\
\hline guaratuba & An 7705 & Pyrogenes & NA & 1 \\
\hline hardjoprajitno. & Hardjoprajitno & Sejroe & L. interrogans & 1 \\
\hline hebdomadis & Hebdomadis & Hebdomadis & L. interrogans & 1 \\
\hline icterohaemorrhagiae & RGA & Icterohaemorrhagiae & L. interrogans & 1 \\
\hline kennewicki & LT 1026 & Pomona & $L$. interrogans & 1 \\
\hline kremastos & Kremastos & Hebdomadis & L. interrogans & 1 \\
\hline mankarso & Mankarso & Icterohaemorrhagiae & L. interrogans & 1 \\
\hline pomona & Pomona & Pomona & L. interrogans & 1 \\
\hline pyrogenes & Salinem & Pyrogenes & L. interrogans & 1 \\
\hline robinsoni & Robinson & Pyrogenes & L. interrogans & 1 \\
\hline swajizak & Swajizak & Mini & NA & 1 \\
\hline valbuzzi & Valbuzzi & Grippotyphosa & NA & 1 \\
\hline zanoni & Zanoni & Pyrogenes & L. interrogans & 1 \\
\hline arborea & Arborea & Ballum & NA & 2 \\
\hline balcanica & 1627 Burgass & Sejroe & L. borgpetersenii & 2 \\
\hline ballum & Mus 127 & Ballum & L. borgpetersenii & 2 \\
\hline hardjobovis & 11135 and $H b-15 b-067$ & Sejroe & L. borgpetersenii & 2 \\
\hline javanica & Bat 46 & Javanica & L. borgpetersenii & 2 \\
\hline kwale & Julu & Pyrogenes & L. borgpetersenii & 2 \\
\hline sejroe & M 84 & Sejroe & L. borgpetersenii & 2 \\
\hline tarassovi & Perepelicin & Tarassovi & L. borgpetersenii & 2 \\
\hline alexi & HS 616 & Pyrogenes & L. interrogans & 3 \\
\hline princestown & TRVL 112499 & Pryogenes & L. santarosai & 3 \\
\hline shermani & $1342 \mathrm{~K}(=\mathrm{LT} 821)$ & Shermani & L. santarosai & 3 \\
\hline varela & 1019 & Pryogenes & L. santarosai & 3 \\
\hline louisiana & LSU 1945 & Louisiana & L. noguchii & 4 \\
\hline myocastoris & LSU 1551 & Pyrogenes & NA & 4 \\
\hline celledoni & Celledoni & Celledoni & L. weilii & 5 \\
\hline sarmin & Sarmin & Sarmin & L. weilii & 5 \\
\hline bulgarica & Nikolaevo & Autumnalis & L. kirschneri & 6 \\
\hline cyanopteri & $3522 \mathrm{C}$ & Cyanopteri & L. kirschneri & 6 \\
\hline grippotyphosa & Moskva V & Grippotyphosa & L. interrogans or $L$. kirschneri & 6 \\
\hline $\begin{array}{l}\text { ranarum } \\
\text { ren }\end{array}$ & ICF & Ranarum & L. meyeri & 7 \\
\hline hamptoni & Hampton & Pyrogenes & NA & 8 \\
\hline
\end{tabular}

NA, not assigned.

${ }^{*}$ Genome species assignments are from Ramadass et al. [10] and Yasuda et al. [11] except for serovars alexi, broomi, camlo, kwale, mankarso, princestown, robinsoni and varela, which were taken from the serovar list published by Faine [14].

reactions. Preliminary experiments demonstrated that consistent profiles could be obtained over a wide range of DNA concentrations (up to 10000-fold; results not shown). Similar results were reported by Welsh and McClelland [2], who attributed the reproducibility of their RAPD profiles to the use of a low annealing temperature in the first few amplification cycles followed by a high annealing temperature for the rest of the cycles.

This method has been used to identify bovine isolates of Leptospira [8]. The fact that almost all serovars examined in this study had unique RAPD profiles strongly suggests that a simplified RAPD such as this could be used for typing isolates from a wide variety of sources. It is possible to design an RAPDprocedure with a predetermined degree of resolution depending on the primer used. For example, primers such as L10 and US were useful for examining similarities between isolates and serovars, perhaps as a tool for making presumptive species assignments. Primer L7 provided finer resolution and was useful for differentiating between closely related serovars and genotypes.

The serovar groupings produced by simplified RAPD agreed with species assignments made by DNA hybridisation $[10,11]$ and those made by Ralph et al. [7] by AP-PCR and other molecular techniques. Serovar groupings by DNA hybridisation analysis [11] did not correspond with serogroups. Similarly, serogroup Pyrogenes reference strains were distributed among five RAPD groups, corresponding to four genome species. The fifth group contained only one member, serovar hamptoni, and did not correlate with any recognised genome species. Serovar hamptoni has 

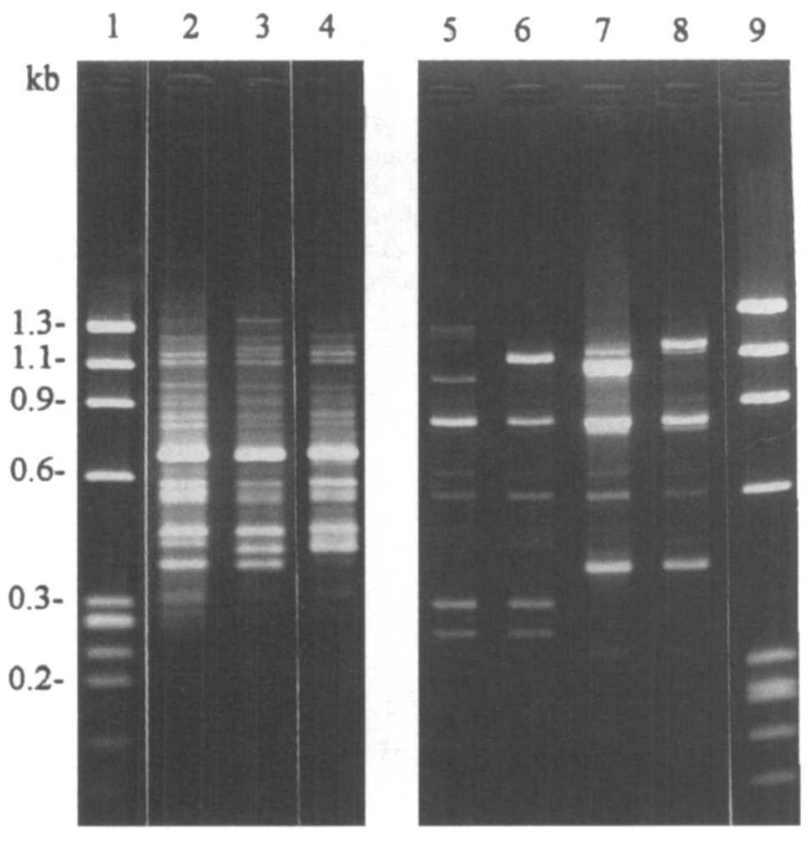

Fig. 3. RAPD profiles of hard-to-resolve serovars obtained by amplification with primer L7. Lanes 1 and 9, $\phi \mathrm{X} 174$ replicative form DNA digested with Hae III; 2, hardjobovis genotype A; 3, hardjobovis genotype B; 4, balcanica; 5 , robinsoni; 6 , zanoni; 7 , pomona; 8 , kennewicki.

not yet been assigned to a genome species. The results of the present study suggest that this serovar may represent an additional genome species. However, Pérolat et al. [16] found similarities in the ribotypes of hamptoni and serovars of $L$. borgpetersenii. DNA hybridisation comparisons of serovar hamptoni with other serovars are necessary to clarify its taxonomic position.

The only serovar for which the RAPD grouping did not correspond with prior genome species assignments was alexi. Faine [14] listed this serovar in $L$. interrogans, whereas this study placed it in RAPD group 3, which corresponds with $L$. santarosai. Similarly, Pérolat et al. [16] reported similarities between the ribotypes of serovar alexi and those of L. santarosai. This serovar was not included in any of the published DNA hybridisation studies $[10,11]$.

Serovars guaratuba, swajizak, valbužzi, arborea and myocastoris are not at present assigned to a genome species. The first three were placed in RAPD group 1, arborea in RAPD group 2 and myocastoris in RAPD group 4 corresponding to $L$. interrogans, L. borgpetersenii and $L$. noguchii, respectively. Previously it was suggested that serovar swajizak be grouped with other $L$. interrogans serovars in a small scale survey of serovars that may be associated with cattle [8].

Ralph et al. [7] queried the validity of $L$. weilii as a single species because of the degree of difference they observed between serovars celledoni and sarmin in AP-PCR and mapped restriction site polymorphism
(MRSP) analysis. In the RAPD analyses, reference strains of these serovars were sufficiently similar to be placed in the same group corresponding to $L$. weilii. This disparity in results probably reflects differences in resolution between primers, similar to differences observed in the performance of L10 and US when compared with L7.

Other workers disagree on the placement of serovar grippotyphosa. Yasuda et al. [11] assigned grippotyphosa to $L$. interrogans, whereas Ramadass et al. [10] assigned the serovar to their newly proposed species L. kirschneri. The RAPD results in the present study and the AP-PCR and MRSP results of Ralph et al. [7] support the assignment of serovar grippotyphosa to $L$. kirschneri.

In conclusion, RAPD coupled with agarose gel electrophoresis is a rapid and simple method for making preliminary genome species assignments. RAPD also has potential as a rapid method for serovar identification.

We thank L. Smythe for critically appraising the manuscript and the staff of the WHO Collaborating Centre for Reference and Research on Leptospirosis, Carole Bolin, and Commonwealth Serum Laboratories for supplying the cultures.

\section{References}

1. Williams JGK, Kubelik AR, Livak KJ, Rafalski JA, Tingey SV. DNA polymorphisms amplified by arbitrary primers are useful as genetic markers. Nucleic Acids Res 1990; 18: 6531-6535.

2. Welsh J, McClelland $M$. Fingerprinting genomes using PCR with arbitrary primers. Nucleic Acids Res 1990; 18: 7213-7218.

3. Brousseau R, Saint-Onge A, Préfontaine G, Masson L, Cabana J. Arbitrary primer polymerase chain reaction, a powerful method to identify Bacillus thuringiensis serovars and strains. Appl Environ Microbiol 1993; 59: 114-119.

4. Jayarao BM, Bassam BJ, Caetano-Anollés G, Gresshoff PM, Oliver SP. Subtyping of Streptococcus uberis by DNA amplification fingerprinting. $J$ Clin Microbiol 1992; 30: 1347-1350.

5. Mazurier S, van de Giessen A, Heuvelman $\mathrm{K}$, Wernars $\mathrm{K}$. RAPD analysis of Campylobacter isolates: DNA fingerprinting without the need to purify DNA. Lett Appl Microbiol 1992; 14: 260-262.

6. Welsh J, Pretzman C, Postic D, Girons IS, Baranton G, McClelland $M$. Genomic fingerprinting by arbitrarily primed polymerase chain reaction resolves Borrelia burgdorferi into three distinct phyletic groups. Int $J$ Syst Bacteriol 1992; 42: 370-377.

7. Ralph D, McClelland M, Welsh J, Baranton G, Perolat $P$. Leptospira species categorized by arbitrarily primed polymerase chain reaction (PCR) and by mapped restriction polymorphisms in PCR-amplified rRNA genes. J Bacteriol 1993; 175: 973-981.

8. Corney BG, Colley J, Djordjevic SP, Whittington R, Graham GC. Rapid identification of some Leptospira isolates from cattle by random amplified polymorphic DNA fingerprinting. J Clin Microbiol 1993; 31: 2927-2932.

9. Pérolat P, Merien F, Ellis WA, Baranton G. Characterization of Leptospira isolates from serovar hardjo by ribotyping, arbitrarily primed PCR, and mapped restriction site polymorphisms. J Clin Microbiol 1994; 32: 1949-1957.

10. Ramadass P, Jarvis BDW, Comer RJ, Penny D, Marshall RB. Genetic characterization of pathogenic Leptospira species by DNA hybridization. Int $J$ Syst Bacteriol 1992; 42: 215-219.

11. Yasuda $\mathbf{P H}$, Steigerwalt AG, Sulzer KR, Kaufmann AF, Rogers 
F, Brenner DJ. Deoxyribonucleic acid relatedness between serogroups and serovars in the family Leptospiraceae with proposals for seven new Leptospira species. Int. J Syst Bacteriol 1987; 37: 407-415.

12. Ramadass $\mathrm{P}$, Jarvis BDW, Corner RJ, Cinco M, Marshall RB. DNA relatedness among strains of Leptospira biflexa. Int $J$ Syst Bacteriol 1990; 40: 231-235.

13. Sambrook J, Fritsch EF, Maniatis T (eds). Molecular cloning: a laboratory manual, 2nd edn. Cold Spring Harbor, NY, Cold Spring Harbor Laboratory Press. 1989.
14. Faine S. Leptospira and leptospirosis. Boca Raton, FL, CRC Press. 1994.

15. Thiermann AB, Handsaker AL, Foley JW, White FH, Kingscote BF. Reclassification of North American leptospiral isolates belonging to serogroups Mini and Sejroe by restriction endonuclease analysis. Am J Vet Res 1986; 47: 61-66.

16. Pérolat P, Lecuyer I, Postic D, Baranton G. Diversity of ribosomal DNA fingerprints of Leptospira serovars provides a database for subtyping and species assignation. Res Microbiol 1993; 144: 5-15. 